Research Paper

\title{
Up-Regulated Lipocalin-2 in Pulmonary Hypertension Involving in Pulmonary Artery SMC Resistance to Apoptosis
}

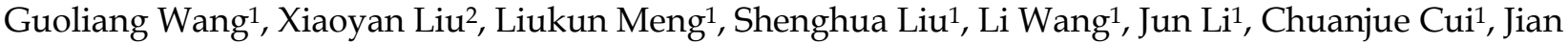

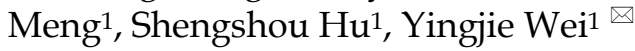 \\ 1. State Key Laboratory of Cardiovascular Disease, Fuwai Hospital, National Center for Cardiovascular Diseases, Chinese Academy of \\ Medical Sciences and Peking Union Medical College, Beijing, 100037, People's Republic of China. \\ 2. Medical Research Center, Beijing Chao-Yang Hospital, Capital Medical University, Beijing, 100020, People's Republic of China.
}

\begin{abstract}
$\square$ Corresponding author: Yingjie Wei, State Key Laboratory of Cardiovascular Disease, Fuwai Hospital, National Center for Cardiovascular Diseases, Chinese Academy of Medical Sciences and Peking Union Medical College, Beijing, 100037, People's Republic of China. Tel: 86-10-60866393. E-mail: weiyingjie@fuwaihospital.org.
\end{abstract}

() Ivyspring International Publisher. This is an open-access article distributed under the terms of the Creative Commons License (http://creativecommons.org/ licenses/by-nc-nd/3.0/). Reproduction is permitted for personal, noncommercial use, provided that the article is in whole, unmodified, and properly cited.

Received: 2014.04.23; Accepted: 2014.06.16; Published: 2014.07.17

\begin{abstract}
A key feature of pulmonary hypertension $(\mathrm{PH})$ is the remodeling of small pulmonary arteries due to abnormal pulmonary artery smooth muscle cell (PASMC) proliferation and resistance to apoptosis. However, the cellular mechanisms underlying how PASMCs in the pathological condition of pulmonary hypertension become resistant to apoptosis remain unknown. It was recently reported that lipocalin 2 (Lcn2) is up-regulated in a wide array of malignant conditions, which facilitates tumorigenesis partly by inhibiting cell apoptosis. In this study, we observed that the expression levels of Lcn2 were significantly elevated in a rat $\mathrm{PH}$ model induced with monocrotaline and in patients with congenital heart disease-associated PH (CHD-PH) when compared with respective control. Therefore, we hypothesize that Lcn2 could regulate human PASMC (HPASMC) apoptosis through a mechanism. By the detection of DNA fragmentation using the TUNEL assay, the detection of Annexin V/PI-positive cells using flow cytometry, and the detection of cleaved caspase- 3 and caspase- 3 activity, we observed that Lcn2 significantly inhibited HPASMC apoptosis induced by serum withdrawal and $\mathrm{H}_{2} \mathrm{O}_{2}$ treatment. We also observed that Lcn2 down-regulated the proapoptotic protein Bax, decreased the levels of cellular ROS, and up-regulated the expression of superoxide dismutases (SODI and SOD2). In conclusion, Lcn2 significantly inhibits HPASMC apoptosis induced by oxidative stress via decreased intracellular ROS and elevated SODs. Up-regulation of Lcn2 in a rat PH model and CHD-PH patients may be involved in the pathological process of $\mathrm{PH}$.
\end{abstract}

Key words: apoptosis, oxidative stress, Lipocalin-2 (Lcn2), SOD, ROS, pulmonary hypertension $(\mathrm{PH})$

\section{Introduction}

Pulmonary hypertension $(\mathrm{PH})$ is a severe disease with poor prognosis. It is characterized by persistent elevated pulmonary arterial pressure (PAP) and ultimately progresses to right ventricular failure and death. A key feature of vascular pathology in $\mathrm{PH}$ is the remodeling of small pulmonary arteries, which results in a persistent elevation of pulmonary arterial resistance [1]. Abnormal pulmonary artery smooth muscle cell (PASMC) proliferation and resistance to apoptosis have been proposed to be essential for vascular remodeling in $\mathrm{PH}[2,3]$. In animal experiments, the induction of apoptosis or coupling with the 
inhibition of the proliferation of PASMCs prevents the progression of medial hypertrophy [4-6]. However, the cellular mechanisms underlying how normal PASMCs become resistant to apoptosis remain unknown.

Increased oxidative stress and elevated levels of reactive oxygen species (ROS) have been observed in animal models of $\mathrm{PH}$ and $\mathrm{PH}$ patients $[7,8]$. ROS play essential roles in the regulation of several physiological and pathological processes, and excessive amounts of ROS are lethal [9]. However, ROS have been demonstrated as important proliferative rather than apoptotic mediators in pulmonary vascular cells $[10,11]$. The mechanisms underlying how increased levels of ROS exert a proliferative effect rather than a proapoptotic effect in $\mathrm{PH}$ remain unclear.

Lipocalin 2 (Lcn2), also known as neutrophil gelatinase-associated lipocalin (NGAL), is a $25-\mathrm{KDa}$ secretary glycoprotein that belongs to the lipocalin family. Lcn2 is an acute phase protein and proinflammatory marker [12-14]. Recent studies have shown that Lcn2 plays a role in cell survival in a wide array of benign and malignant conditions. It has been reported as a survival factor and can protect cells against apoptosis [15-18]. Lcn2 is over-expressed in cancers of diverse histological origin and it facilitates tumorigenesis by promoting survival, growth, and metastasis $[18,19]$. Paradoxically, Lcn2 induces apoptosis in other conditions. For example, Lcn2 can induce the apoptosis of H9c2 cardiomyocytes [20] and A549 human lung adenocarcinoma cells [21]. Thus, Lcn2 appears to be a double-edged sword in apoptosis and cell survival.

The up-regulation of Lcn2 in malignant conditions and its roles in cell survival motivated us to examine whether Lcn2 is over-expressed and its effect on HPASMC apoptosis, which is an important component of small pulmonary artery remodeling. Remarkably, prior to this paper, there has been no report on the role of Lcn2 in $\mathrm{PH}$ and in the survival of PASMCs. Because ectopically expressed Lcn2 has been reported to induce the expression of heme oxygenase-1,2 and SOD1,2 in CHO cells [17], we hypothesized that the up-regulation of antioxidant enzymes is an important mechanism in Lcn2-inhibited HPASMC apoptosis. Thus, this study aimed to determine whether Lcn 2 expression is altered in a rat $\mathrm{PH}$ model induced with monocrotaline and in patients with congenital heart disease associated PH (a large subtype of $\mathrm{PH}$ ) and to analyze the role of Lcn2 on HPASMC apoptosis at the cellular and molecular levels, which may help us better understand the pathophysiological role of Lcn2 in $\mathrm{PH}$.

\section{Methods}

\section{Animal model of pulmonary hypertension}

All protocols and surgical procedures were approved by the local animal care committee. Adult male Sprague-Dawley rats (6 to 7 weeks of age; weight 250 to $300 \mathrm{~g}$ ) were administered a single subcutaneous injection of $60 \mathrm{mg} / \mathrm{kg}$ monocrotaline (MCT) or saline. The animals were killed 7, 14, 21, 28 and 35 days after the subcutaneous injection of MCT or saline, the body weight was recorded, and right-heart catheterization was performed to measure the pulmonary arterial pressure. The rats were exsanguinated immediately after hemodynamic assessment, and the lungs were removed for further analysis. A part of the lungs from all of the groups were fixed in situ in the distended state by infusion of $10 \%$ neutral buffered formalin into the pulmonary artery (at $25 \mathrm{~mm} \mathrm{Hg}$ pressure) and trachea for $1 \mathrm{mi}$ nute and then placed in $10 \%$ neutral buffered formalin at $4^{\circ} \mathrm{C}$ for $24 \mathrm{~h}$ prior to embedment in paraffin. The remaining lungs were immediately frozen in liquid nitrogen for protein and RNA isolation. To assess the extent of $\mathrm{RVH}$, the heart was removed, and the $\mathrm{RV}$-free wall was dissected from the left ventricle plus septum (LV+S) and weighed separately; the degree of right ventricular hypertrophy (RVHI) was determined from the ratio $R V /(\mathrm{LV}+\mathrm{S})$, as previously described [22].

\section{Patients and sampling}

This study was approved by the ethics committee at Fuwai Hospital and conforms to the principles outlined in the Declaration of Helsinki. Informed consent was obtained from the parents of all of the patients. Patients with congenital heart disease-associated pulmonary hypertension (CHD-PH, measured through echocardiography) aged 6 to 24 months who underwent a surgical correction were included in the study. Patients with congenital heart diseases with unrestricted left-to-right shunt but no PH (CHD-nonPH) aged 6 to 24 months, who underwent surgical correction, were also included in the study as control subjects. Patients with any other diseases or clinical signs that were not associated with congenital heart diseases were excluded. Citrated plasma samples were obtained and stored in aliquots at $-70{ }^{\circ} \mathrm{C}$. The level of plasma Lcn2 was measured using the Human Lipocalin-2/NGAL Quantikine ELISA Kit (DLCN20, R\&D, USA) according to the manufacturer's instructions.

\section{Immunohistochemistry}

Immunostaining for Lcn2 was performed in the lungs of rats after 3 weeks of exposure to mono- 
crotaline or saline. The lung samples were sectioned at 5- $\mu \mathrm{m}$ thickness. Rabbit polyclonal anti-Lcn2 (Santa Cruz Biotechnology, USA) was detected using the Strept ABC peroxidase technique (ZSGB-BIO, CHN) as previously described [23].

\section{Cell culture}

Human pulmonary artery SMCs were purchased from ScienCell (USA) and used according to the manufacturer's instructions or maintained in DMEM supplemented with 10\% fetal bovine serum (FBS), $1 \%$ penicillin / streptomycin at $5 \% \mathrm{CO}_{2}$ and $37^{\circ} \mathrm{C}$ as previously described [23]. The cells were starved for 24 hours in $0.1 \%$ FBS-containing medium prior to treatment with recombinant human Lcn2 (RayBiotech, USA) or $\mathrm{H}_{2} \mathrm{O}_{2}$ (Beijing Chemical Works, $\mathrm{CHN}$ ) at the indicated concentrations.

\section{Measurements of Apoptosis}

The apoptotic cells were quantified using the terminal deoxyribonucleotide transferase-mediated dUTP nick end-labeling (TUNEL) assay (In Situ Cell Death Detection Kit, KeyGEN BioTECH, CHN) according to the manufacturer's instructions. Fluorescent staining was visualized using a BX-61 microscope (Olympus). To further quantify the number of apoptotic cells, flow cytometry (ACCURY C6, BD, USA) using the FITC Annexin V Apoptosis Detection Kit (KeyGEN BioTECH, CHN) was performed according to the manufacturer's instructions, as previously described [23]. Caspase-3 activity was measured using a Caspase-3 Activity Assay Kit (Beyotime, CHN) according to the manufacturer's instructions.

\section{Quantitative real-time RT-PCR}

Frozen rat lung tissue was homogenized with the Trizol reagent (Invitrogen, USA), and the total RNA was extracted. Cells grown in $25-\mathrm{cm}^{2}$ flasks or 6-well plates were washed with PBS and lysed directly in the culture dish with the Trizol reagent. The total RNA was extracted and reverse-transcribed using Oligo (dT) and Transcriptor Reverse Transcriptase (Roche, GA) according to the manufacturer's instructions. The gene transcripts were quantified by real-time quantitative PCR (qPCR) using an Applied Biosystems 7300 Fast Real-Time PCR System (ABI, USA) with SYBR Green PCR Master Mix, as previously described [23]. The expression of gene transcripts in the test samples was normalized against the internal standard glyceraldehyde-3-phosphate dehydrogenase (GAPDH). The primers used are listed in Table 1.

\section{Western blotting analyses}

Frozen lung tissue was homogenized in RIPA lysis buffer (Beyotime, CHN) and centrifuged for 10 minutes at $15,000 \times g$ and $4^{\circ} \mathrm{C}$. The cells were trypsinized, washed twice with PBS, lysed with RIPA lysis buffer on ice for at least $30 \mathrm{~min}$, and centrifuged for 10 minutes at $15,000 \times g$ and $4^{\circ} \mathrm{C}$. The protein concentration was determined using the BCA Protein Assay Kit (Beyotime, CHN) with BSA as the standard. An equal amount of protein $(50 \mu \mathrm{g}$ for rat lung samples, $20 \mu \mathrm{g}$ for cell samples) from each sample was diluted with $5 \times$ sample loading buffer and boiled for 5 minutes. The protein suspensions were separated on $12 \%$ SDS-PAGE gel and transferred onto a nitrocellulose membrane, and the proteins were detected using the following antibodies: rabbit anti-Lcn2, rabbit anti-cleaved caspase-3, rabbit anti-SOD1, rabbit anti-SOD2, rabbit anti-Bax (from Santa Cruz Biotechnology, USA), rabbit anti-Bcl-2 (from RayBiotech, GA), mouse anti-GAPDH, goat anti-rabbit IgG, and goat anti-mouse IgG (from ZSGB-BIO, CHN).

Table I. Primers used for Real-Time RT-PCR

\begin{tabular}{ll}
\hline Rat GAPDH forward & 5'-GGCACAGTCAAGGCTGAGAATG-3' \\
\hline Rat GAPDH reverse & 5'-ATGGTGGTGAAGACGCCAGTA-3' \\
Rat Len2 forward & 5'-TTCCATCCTCGTCAGGGGCCA-3' \\
Rat Lcn2 reverse & 5'-CGGCCACTTGCACATCGTAGC-3' \\
Human GAPDH forward & 5'-GCACCGTCAAGGCTGAGAAC-3' \\
Human GAPDH reverse & 5'-TGGTGAAGACGCCAGTGGA-3' \\
Human SOD1 forward & 5'-TTTGCGTCGTAGTCTCCTGC-3' \\
Human SOD1 reverse & 5'-CCACACCTTCACTGGTCCAT-3' \\
Human SOD2 forward & 5'-GTTGGGGTTGGCTTGGTTTC-3' \\
Human SOD2 reverse & 5'-TGCTCCCACACATCAATCCC-3' \\
Human Bax forward & 5'-CCAAGAAGCTGAGCGAGTGT-3' \\
Human Bax reverse & 5'-GGAAAAAGACCTCTCGGGGG-3' \\
\hline
\end{tabular}

\section{Determination of reactive oxygen species [24]}

The ROS levels were measured using the Reactive Oxygen Species Assay Kit (Beyotime, CHN) according to the manufacturer's instructions. A membrane-permeable fluorogenic probe, dichlorodihydrofluorescein diacetate (DCFDA), was used. The acetate and acetoxymethyl ester groups of this probe were enzymatically cleaved inside living cells. This probe can then be oxidized by intracellular oxidants (ROS) to obtain the product DCF, which emits a strong green fluorescence $\left(\lambda_{\mathrm{ex}}=485 \mathrm{~nm} ; \lambda_{\mathrm{em}}=520 \mathrm{~nm}\right)$. The fluorescence intensity is proportional to the level of cellular ROS. After the indicated treatment, cells grown in a 6-well plate were loaded in PBS containing $10 \mu \mathrm{M}$ DCFDA fluorescent probe at $37^{\circ} \mathrm{C}$ for $20 \mathrm{~min}$ and then washed 3 times with PBS and trypsinized. The cells were obtained after centrifugation at $200 \times g$ for $5 \mathrm{~min}$ at $4^{\circ} \mathrm{C}$, resuspended and incubated in ice-cold PBS. The fluorescence of the cells was monitored using flow cytometry (ACCURY C6, BD, USA). ROS production was calculated as the fold increase in 
the fluorescence compared with that observed in the control.

\section{Statistical analyses}

Each independent experiment was repeated at least three times, and at least 3 independent experiments were performed. The data are shown as the means $\pm S E$. Student's t-test was used for comparisons between two groups, and $p<0.05$ was considered significant.

\section{Results}

\section{Increased expression of Lcn 2 in rat $\mathrm{PH}$ model and in patients with CHD-PH}

We used a monocrotaline-induced rat $\mathrm{PH}$ model to determine whether Lcn2 expression is altered in $\mathrm{PH}$. Three weeks after the MCT injection, the hemodynamic assessment showed that MCT significantly enhanced the pulmonary arterial pressure (Supplementary Material: Fig. S1A) and resulted in significant right ventricular hypertrophy (Supplementary Material: Fig. S1B). The rat lung samples were collected to examine the expression level of Lcn2. Both western blotting analyses (Fig. 1A) and real time-PCR (Fig. 1B) revealed that the expression levels of Lcn2 were significantly increased after MCT injection. The protein levels increased significantly as early as 7 days after MCT injection (Fig. 1A). Immunostaining for Lcn2 also showed its up-regulation in the lungs of rats 3 weeks after MCT injection (Fig. 1C). Consistent with our cellular results obtained from HPASMCs (data not shown), the immunostaining analyses of Lcn2 in rat PASMCs showed undetectable expression.

We then examined the Lcn2 levels in $\mathrm{PH}$ patients. Twenty-two patients with congenital heart disease were included in the study and divided into 2 groups: $10 \mathrm{CHD}-\mathrm{PH}$ patients (age $13.0 \pm 3.7$ months, $60 \%$ males) and $12 \mathrm{CHD}$-nonPH patients (age $12.8 \pm$ 4.4 months, $50 \%$ males). The plasma samples from patients with $\mathrm{CHD}-\mathrm{PH}$ and $\mathrm{CHD}-n o n \mathrm{PH}$ were subjected to immunoblotting. As shown in Fig. 2, the $\mathrm{CHD}-\mathrm{PH}$ patients exhibited significantly higher plasma Lcn2 concentrations compared with the CHD-nonPH patients.
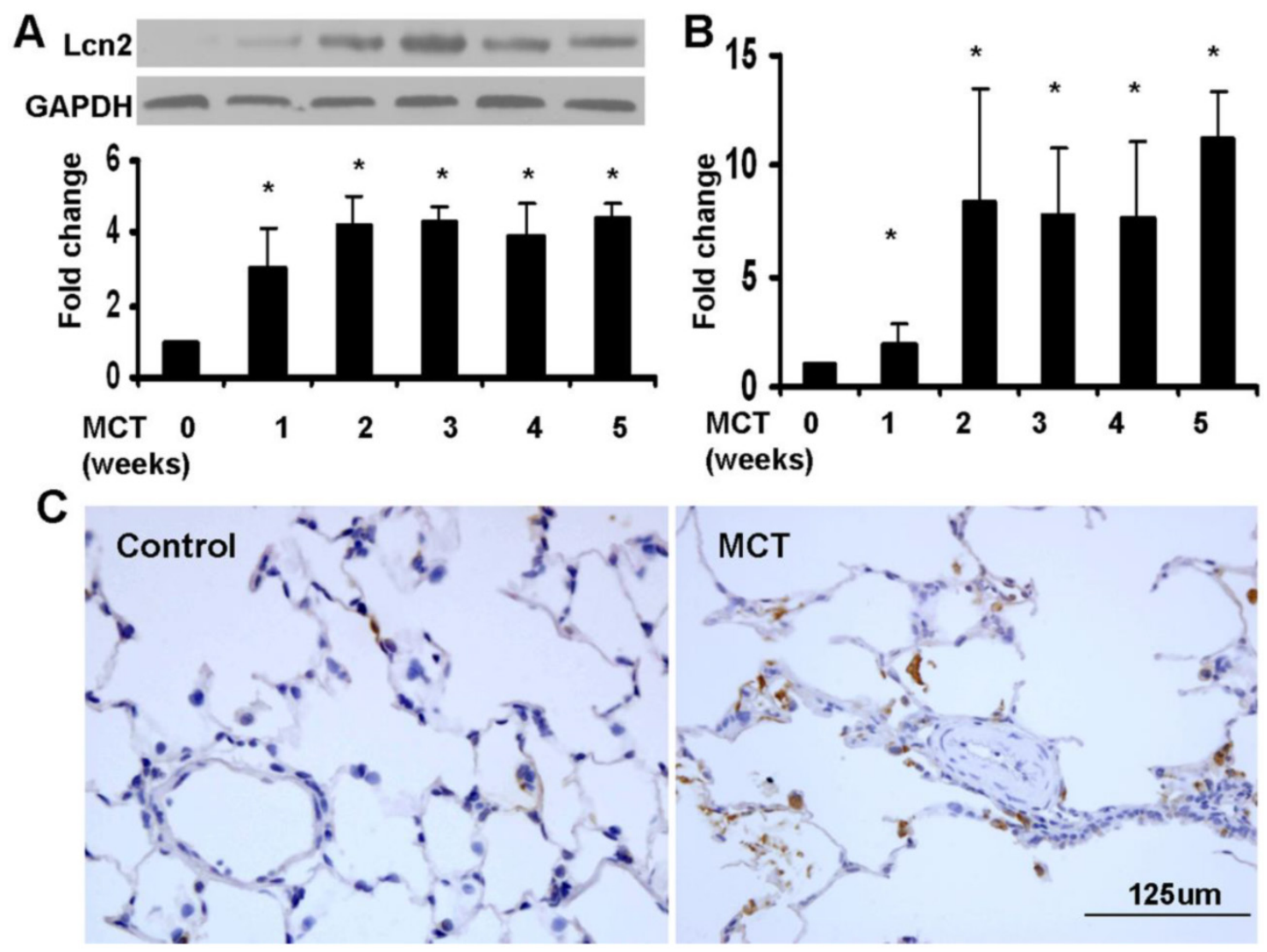

Figure I. The level of Lcn2 in rat PH model induced with MCT. 7, I4, 2 I, 28 and 35 days after the subcutaneous injection of MCT or saline, the lung samples of rats were collected. A, Total protein was extracted and subjected to Western blotting analyses. Representative western blot and quantitative analysis are shown ( $\mathrm{n}=6$; $*$, $p<0.05$ compared with control). B, Total RNA was extracted and Real-time PCR for Lcn 2 was performed ( $n=6$; ${ }^{*}, p<0.05$ compared with control). C, Immunostaining for LCN2 in lungs of rat after 3 weeks of MCT (or saline) injection (400x). 


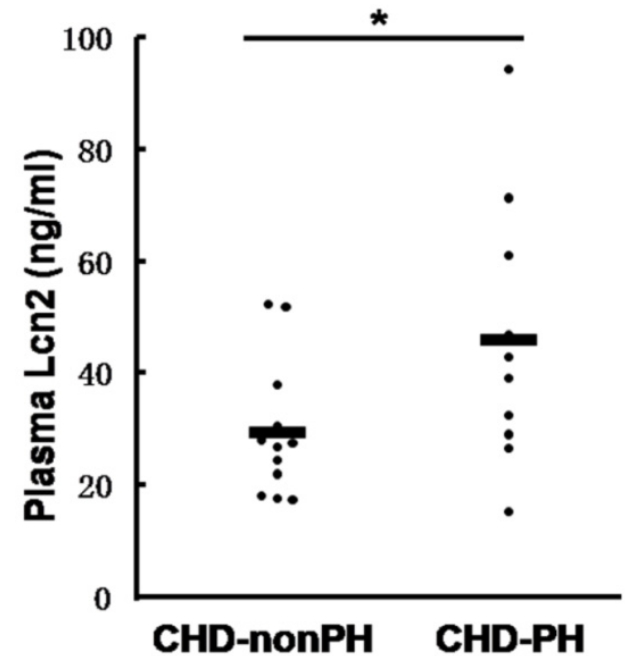

Figure 2. The levels of Len2 in patients with CHD-PH and CHD-nonPH. Plasma samples from patients with CHD-PH $(n=10$, age $13.0 \pm 3.7$ months, $60 \%$ males) and CHD-nonPH ( $n=12$, age $12.8 \pm 4.4$ months, $50 \%$ males) were collected. The level of Lcn2 was measured using the Human Lipocalin-2/NGAL Quantikine ELISA Kit. Heavy lines represent mean value of each group $(*, p<0.05)$.

\section{Len2 inhibits HPASMC apoptosis}

Under conditions of serum deprivation, a few cells will undergo apoptosis due to a lack of survival factors [25]. We used the TUNEL assay to detect DNA fragmentation and found that less apoptotic cells were observed after treatment of HPASMCs with $10 \mathrm{ng} / \mathrm{ml}$ recombinant human Lcn 2 for $24 \mathrm{~h}$ compared with the serum-free medium alone (control). Furthermore, 100 $\mu \mathrm{M} \mathrm{H}_{2} \mathrm{O}_{2}$ induced nearly half of the HPASMCs to undergo apoptosis. In the presence of $10 \mathrm{ng} / \mathrm{ml} \mathrm{Lcn} 2$, the sensitivity of HPASMCs to $\mathrm{H}_{2} \mathrm{O}_{2}$ was decreased (Fig. 3A). The quantitative analysis of TUNELpositive cells demonstrated a significant decrease in apoptotic cells in the Lcn2-treated groups compared with the no-Lcn2-treated groups (Fig. 3B). The role of Lcn2 in HPASMC apoptosis was analyzed further using Annexin V (AV) and propidium iodide (PI) double staining followed by flow cytometry (Fig. 3C). A significant decrease in the population of $\mathrm{AV}^{+} / \mathrm{PI}^{+}$ cells was observed in the Lcn2-treated groups (Fig. 3D).
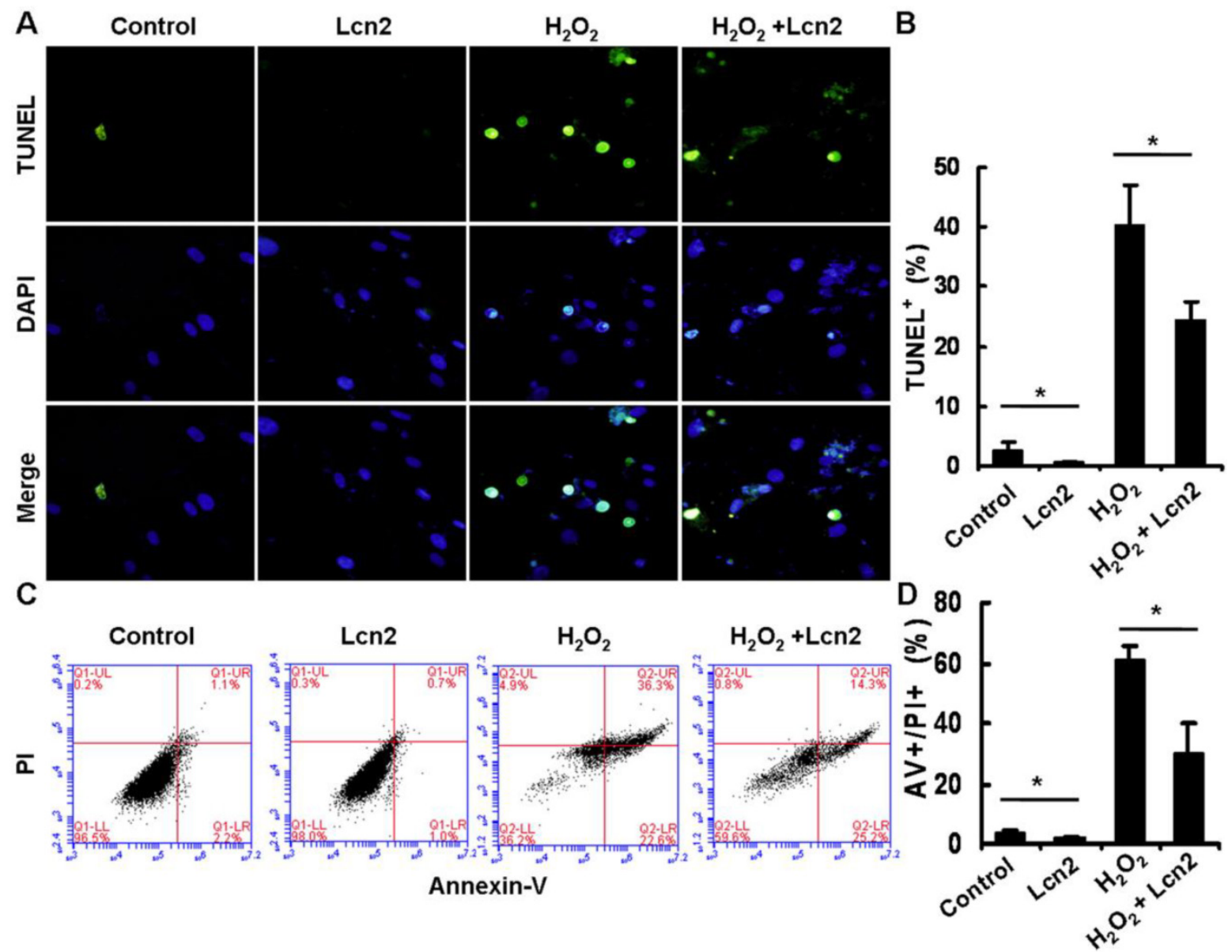

Figure 3. Determination of apoptotic cells using TUNEL assay and flow cytometry. HPASMCs were treated with serum free medium or I00 $\mu M \mathrm{H}_{2} \mathrm{O}_{2}$, in the presence or absence of $10 \mathrm{ng} / \mathrm{ml} \mathrm{Lcn} 2$ for $24 \mathrm{~h}$. A, Apoptotic cells were examined using TUNEL assay for DNA fragmentation. TUNEL-positive nuclei are shown in green, total nuclei stained with DAPI in blue. HPASMCs were photographed by fluorescence microscopy (magnification, $\times 400$ ). B, Quantitative analysis (\% of apoptotic cells versus total, at lease 50 cells per group per experiment) from five randomly selected fields in each individual experiment are shown in histogram ( $n=3 ; *, p<0.05$ ). C, After double staining of HPASMCs, the $\mathrm{AV}^{+}$and $\mathrm{Pl}^{+}$cells were analyzed by flow cytometry (total 20,000 events per group). D, Quantitative determination of data from FACS analysis showing treatment of $\operatorname{Lcn} 2(10 \mathrm{ng} / \mathrm{ml})$ for $24 \mathrm{~h}$ produced a significant decrease in the number of cells undergoing apoptosis $\left(\mathrm{AV}^{+} / \mathrm{PI}^{+}\right)$in both groups $(\mathrm{n}=3 ; *, p<0.05)$. 
Next, we investigated whether caspase-3 cleavage was inhibited by Lcn 2 in HPASMCs. The cells were cultured in serum-free conditions (control) or treated with $100 \mu \mathrm{M} \mathrm{H}_{2} \mathrm{O}_{2}$, in the presence or absence of $10 \mathrm{ng} / \mathrm{ml}$ recombinant human Lcn2 for $24 \mathrm{~h}$. Lcn2-inhibited caspase-3 cleavage was confirmed in both conditions (with or without $\mathrm{H}_{2} \mathrm{O}_{2}$ ) using western blotting analyses (Fig. 4A). We observed that the levels of the cleaved form of caspase-3 $(17 \mathrm{kDa})$ were significantly decreased in the Lcn2-treated groups (Fig. 4A). Next, we directly measured the activity of caspase- 3 using an assay based on the cleavage of a fluorogenic substrate and found that the activity of caspase- 3 was significantly decreased in cells exposed to Len2 (Fig. 4B).

\section{Lcn2 decreases the expression of Bax in HPASMCs}

Bax is an important proapoptotic factor involved in the intrinsic pathway of apoptosis. Bax increases mitochondrial membrane permeability by translocating and integrating into the membrane, thereby promoting mitochondria-dependent apoptosis. The effect of Lcn2 on the expression of this protein was examined. RT-PCR (Fig. 5A) and western blotting analyses (Fig. 5B) revealed that the expression levels of Bax in HPASMCs were significantly decreased in the Lcn2-treated groups. We also examined mitochondria membrane-integrated Bax using an antibody against an epitope in the N-terminal domain of Bax, which is normally obscured and is only exposed when Bax changes its conformation as it integrates into the mitochondrial membrane [26]. A decrease in the endogenous active form of $\mathrm{Bax}$ in the Lcn2-treated group was observed compared with the $\mathrm{H}_{2} \mathrm{O}_{2}$-treated group (Supplementary Material: Fig. S2).

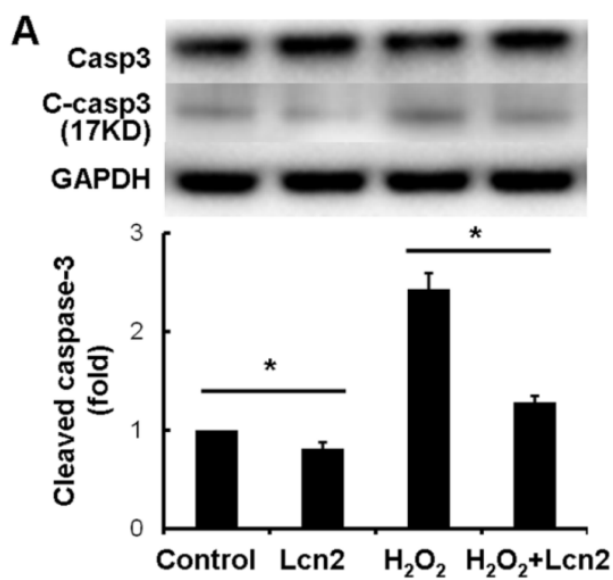

B

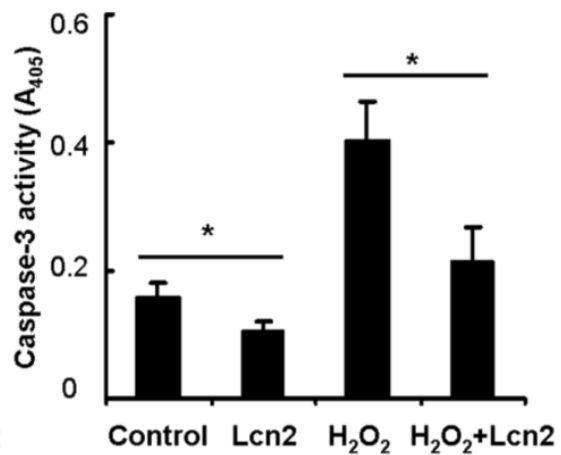

Figure 4. Detection of cleaved caspase-3 and its activity. HPASMCs were treated with serum free medium or $100 \mu M \mathrm{H}_{2} \mathrm{O}_{2}$, in the presence or absence of $\mathrm{I} 0 \mathrm{ng} / \mathrm{ml} \mathrm{Lcn} 2$ for $24 \mathrm{~h}$, total protein was extracted and subjected to Western blotting analyses. A, A representative blot and $\mathrm{n}=3$ independent experiments are quantitated $(*, p<0.05)$. B, Cell lysates were assayed for caspase-3 activity and normalized to total protein concentration, the value of absorbance at $405 \mathrm{~nm}$ is shown $(n=3 ; *, p<0.05)$.
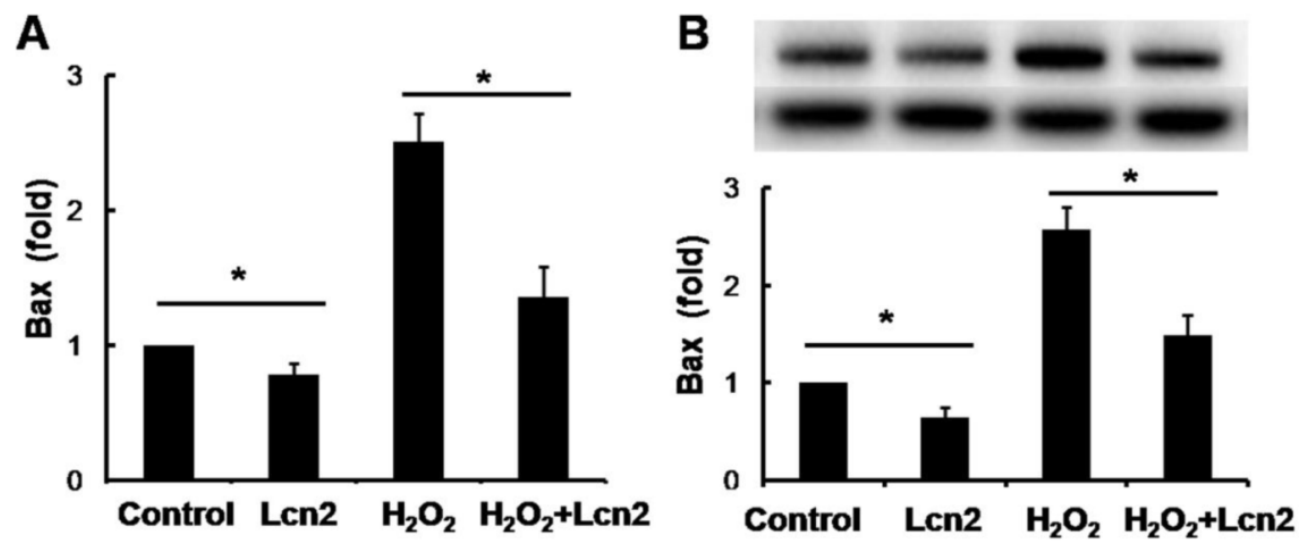

Figure 5. Lcn2 decreases Bax expression. HPASMCs were treated with serum free medium or $100 \mu M \mathrm{H}_{2} \mathrm{O}_{2}$, in the presence or absence of $10 \mathrm{ng} / \mathrm{ml} \mathrm{Lcn} 2 \mathrm{for} 24 \mathrm{~h}$. A, Total RNA was extracted and reverse transcripted to CDNA, quantitative determination of Bax expression using real-time PCR, and normalized against the internal standard GAPDH. $B$, Total protein was extracted and subjected to Western blotting analyses. A representative blot and quantitative analysis are shown. $(n=3 ; *, p<0.05)$. 


\section{Lcn2 decreases intracellular ROS and increases SODI and SOD2 expression}

ROS are important effectors in oxidative stress-induced apoptosis, and excessive amounts of ROS are lethal [9]. Thus, we used the general ROS indicator DCFDA to determine the level of intracellular ROS. The cells were cultured in serum-free conditions (control) or treated with $100 \mu \mathrm{M} \mathrm{H}_{2} \mathrm{O}_{2}$, in the presence or absence of $10 \mathrm{ng} / \mathrm{ml}$ recombinant human Lcn 2 for $12 \mathrm{~h}$. Lcn2 significantly decreased the observed fluorescence in both conditions (with or without $\mathrm{H}_{2} \mathrm{O}_{2}$ ) compared with the no-Lcn2-treated groups (Fig. 6). Superoxide dismutases (SOD1 and SOD2) are major antioxidant enzymes that act against ROS-mediated damage in cells [17]. The effects of Lcn 2 on SOD1 and SOD2 expression were also examined using RT-PCR and western blotting analyses. Lcn2 significantly up-regulated the expression of SOD1 and SOD2 in both conditions (with or without $\mathrm{H}_{2} \mathrm{O}_{2}$ ) compared with the no-Lcn2-treated groups (Fig. 7A). The promoting effects of Lcn2 on SOD1 and SOD2 expression were dose-dependent (Fig. 7B).

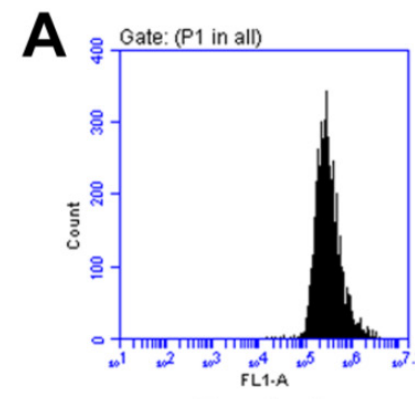

Control

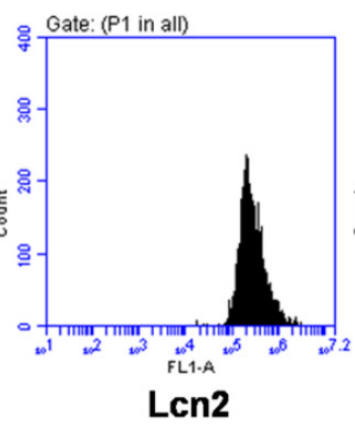

Len2
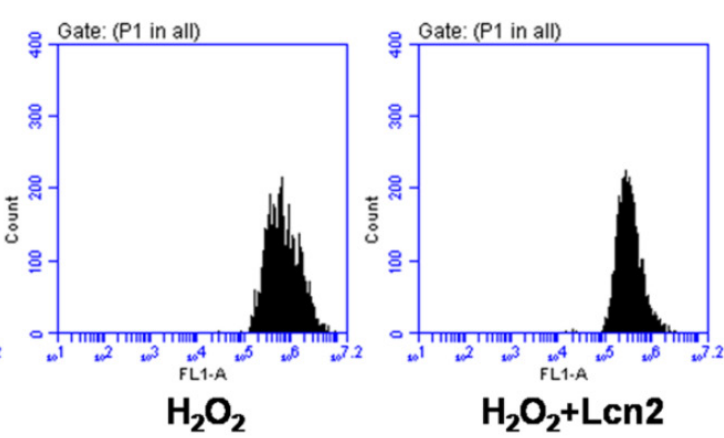

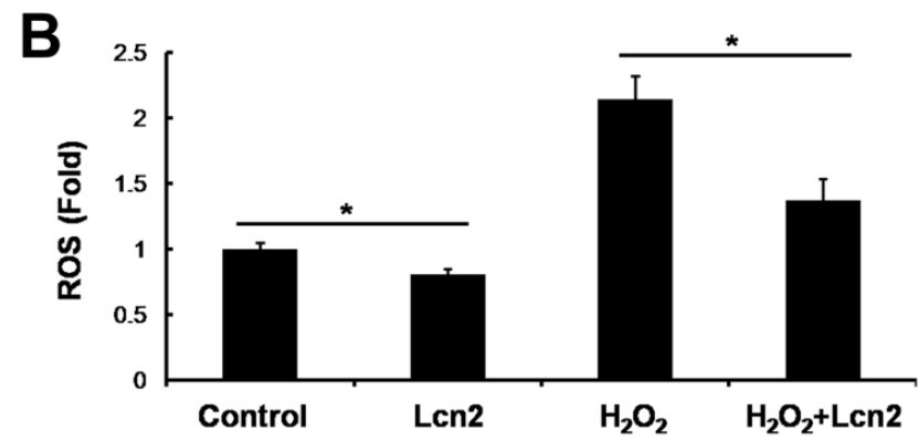

Figure 6. Lcn2 decreases the level of intracellular ROS. HPASMCs were treated with serum free medium or $100 \mu M \mathrm{H}_{2} \mathrm{O}_{2}$, in the presence or absence of $10 \mathrm{ng} / \mathrm{ml} \mathrm{Lcn} 2$ for $24 \mathrm{~h}$, then loaded in PBS containing $10 \mu \mathrm{M}$ DCFDA fluorescent probe at $37^{\circ} \mathrm{C}$ for $20 \mathrm{~min}$. Wash and incubate the cells in ice-cold PBS after tripsinized, the fluorescence of the cells was monitored using flow cytometry. Representative dot plots $(A)$ and quantitative analysis of mean fluorescent intensity $(B)$ are shown $(n=3 ; *, p<0.05)$.
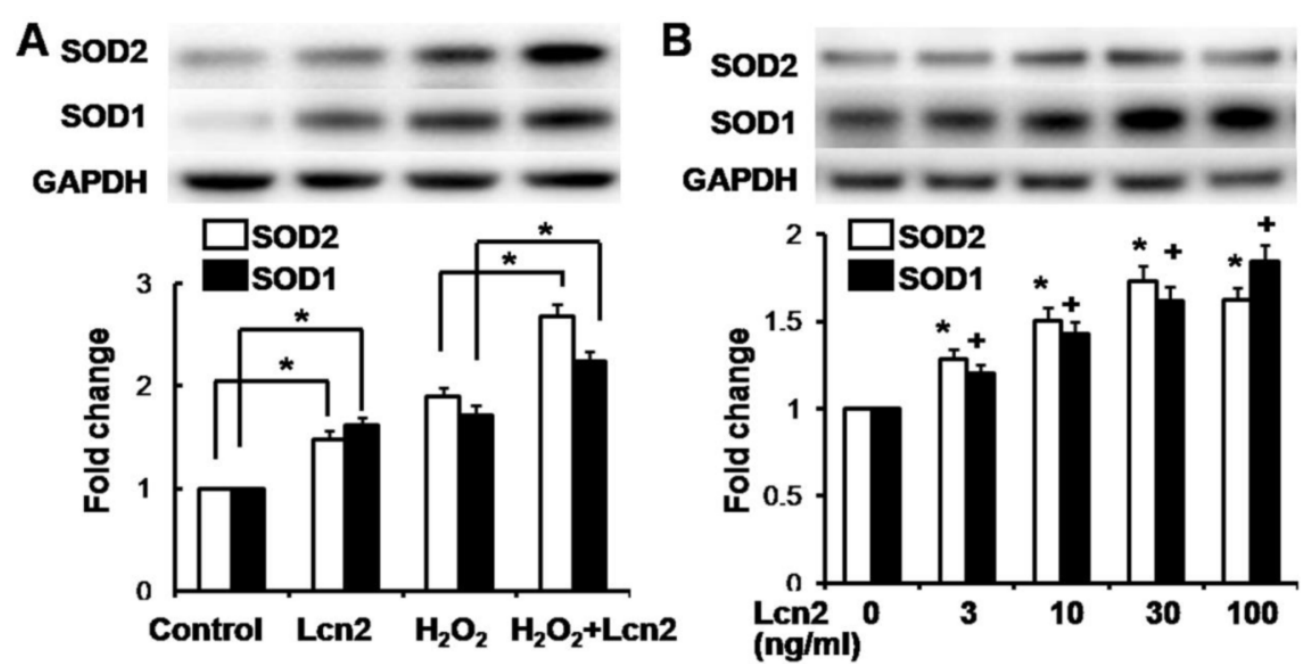

Figure 7. Lcn2 increases the expression of SODI and SOD2. A, HPASMCs were treated with serum free medium or $100 \mu M \mathrm{H}_{2} \mathrm{O}_{2}$, in the presence or absence of 10 $\mathrm{ng} / \mathrm{ml} \mathrm{Lcn} 2$ for $24 \mathrm{~h}$. Total protein was extracted and subjected to Western blotting analyses. A representative blot and quantitative analysis is shown ( $\mathrm{n}=3$; *, $p<0.05$ ). $\mathrm{B}$, HPASMCs were treated with $0,3,10,30$ and $100 \mathrm{ng} / \mathrm{ml} \mathrm{Lcn} 2$ in serum free medium for $24 \mathrm{~h}$, total protein was extracted and subjected to Western blotting analyses. A representative blot and quantitative analysis are shown. $(\mathrm{n}=3 ; *, p<0.05$ compared with SOD2 control; +, $p<0.05$ compared with SODI control). 


\section{Discussion}

Lcn2 was initially shown to combat bacterial infection via the impedance of bacterial iron sequestration [14]. Its small size and secreted nature motivated its investigation as a diagnostic and prognostic biomarker in numerous diseases, including inflammation and cancer [27]. Recent studies have shown that Lcn2 is over-expressed in cancers of diverse histological origin and it facilitates tumorigenesis by promoting survival, growth, and metastasis $[18,19]$, indicating a pivotal role of this molecule in several physiological and pathological conditions. Conflicting results have been reported regarding the role of Lcn2 in cell apoptosis. On the one hand, Lcn2 can protect a cell from apoptosis induced by cellular stress, such as $\mathrm{H}_{2} \mathrm{O}_{2}$ [15-18]. On the other hand, Lcn2 can induce cell apoptosis under specific conditions [20,21]. This motivated us to examine the effect of Lcn2 on HPASMC apoptosis, an important component of small pulmonary arteries remodeling, which results in PH. In this study, we examined the up-regulated expression of Lcn2 in the lungs of rats treated with MCT (Fig. 1). The plasma levels of Lcn2 in CHD-PH patients were also significantly elevated compared with the CHD-nonPH subjects (Fig. 2). These results enhanced the probability that Lcn 2 may play a role in $\mathrm{PH}$.

Using TUNEL staining and flow cytometry analysis, we demonstrated that recombinant Lcn2 can protect HPASMCs from apoptosis induced by serum withdrawal and $\mathrm{H}_{2} \mathrm{O}_{2}$ (Fig. 3). This novel observation was confirmed using measurements of cleaved caspase-3 and its activity (Fig. 4). Although Lcn2 can protect cells against apoptosis, little is known regarding its intracellular mechanisms, particularly in HPASMCs. Thus, we investigated the expression and translocation of Bax, which is involved in the promotion of mitochondria-dependent apoptosis [26]. The expression of Bax was inhibited by Lcn 2 at both the protein and mRNA levels (Fig. 5). In particular, Lcn2 inhibited Bax translocation and integration into the mitochondrial membrane, an important step in the activation of mitochondria-dependent apoptosis, as assessed using an antibody against the conformationally changed form of Bax (Supplementary Material: Fig. S2).

ROS are important effectors in oxidative stress-induced apoptosis, and oxidative damage to macromolecules is mediated by intracellular ROS. Our results confirmed decreased levels of intracellular ROS in the Lcn2-treated groups (Fig. 6). Antioxidant enzymes play a critical role in the regulation of intracellular oxidative stress [17]. Superoxide dismutases (SODs) are important antioxidant enzymes, and an increased amount of SODs reduces the intracellular
ROS levels. In this study, we found that Lcn2 up-regulates the expression of SOD1 and SOD2 at both the transcriptional and translational levels (Fig. $7)$. These findings were consistent with the report of Parisa et al. [17], who found that ectopically expressed $\mathrm{Lcn} 2$ is a protective factor against oxidative stress via the induction of heme oxygenase-1,2 and SOD1,2 expression in $\mathrm{CHO}$ cells. However, the induction of Lcn2 on the expression of heme oxygenase was undetectable in this study (data not shown). Roudkenar et al. [15] reported that Lcn2 exerts its cytoprotective effect independent of HO-1. These reports and our results suggest that SOD1,2 play important roles, at least partially, in Lcn2-inhibited HPASMC apoptosis.

ROS play an essential role in the regulation of several physiological and pathological processes. Moderate increases in ROS can promote cell proliferation [28], whereas extremely excessive amounts of ROS are lethal [9]. Patients with PH are characterized by increased oxidative stress [7]. Elevated levels of ROS are also observed in animal models of PH [8]. ROS have been demonstrated as important proliferative mediators in pulmonary vascular cells. However, whether elevated Lcn2 levels in $\mathrm{PH}$ protect pulmonary vascular cells from apoptosis caused by potentially excessive amounts of ROS and maintains the proliferative effect of ROS by up-regulating SODs deserves further study. Indeed, inducing the apoptosis of hypertrophied PASMCs in animal studies prevents the progression of $\mathrm{PH}$ [18].

Lcn2 has been investigated as a diagnostic and prognostic biomarker in numerous diseases [27]. It is expressed at very low concentrations in healthy children and is markedly elevated in the case of epithelial damage [29,30]. Fadel et al. [31] reported that plasma Lcn2 can be used as an early biomarker for the diagnosis of acute kidney injury following cardio-pulmonary bypass in pediatric cardiac surgery. In this study, the plasma Lcn2 levels in CHD-PH children were significantly elevated compared with those found in CHD-nonPH subjects (Fig. 1). Recent studies have reported that pulmonary vascular remodeling caused by an abnormal hemodynamic state plays an important role in the development of congenital heart disease-associated $\mathrm{PH}[32,33]$. Left-to-right shunt is an important characteristic of congenital heart disease-associated PH. Under higher oxygen saturation and the shear stress of increased blood flow, pulmonary vascular endothelial cells may be damaged and induced to release inflammatory cytokines, which results in inflammatory cell infiltration [34]. This may be the reason why the plasma Lcn2 level was elevated in CHD-PH, which suggests a more serious hemodynamic state than CHD-nonPH. From this perspective, it is possible that the plasma Lcn2 level may be a 
new biomarker of $\mathrm{PH}$, at least for CHD-PH. Further experiments are required to understand the in vivo roles of Lcn2 in PH.

\section{Supplementary Material}

Figures S1 and S2.

http://www.ijbs.com/v10p0798s1.pdf

\section{Acknowledgments}

This work was supported by research grants from the National 973 Program of China (grant 2010CB529505 to SS.H.). We thank Dr. Yu Nie for his valuable suggestions in preparation of the manuscript.

\section{Competing Interests}

The authors have declared that no competing interest exists.

\section{References}

1. Runo JR, Loyd JE. Primary pulmonary hypertension. Lancet 2003; 361:1533-1544

2. Burg ED, Remillard CV, Yuan JX. Potassium channels in the regulation of pulmonary artery smooth muscle cell proliferation and apoptosis: Pharmacotherapeutic implications. Br J Pharmacol 2008;153(Suppl 1):S99-S111.

3. Perros F, Montani D, Dorfmuller P, Durand-Gasselin I, Tcherakian C, Le Pavec $\mathrm{J}$, et al. Platelet-derived growth factor expression and function in idiopathic pulmonary arterial hypertension. Am J Respir Crit Care Med 2008;178(1):81-88.

4. Rajagopalan N, Simon MA, Suffoletto MS, Shah H, Edelman K, Ma thier MA, et al. Noninvasive estimation of pulmonary vascular resistance in pulmonary hypertension. Echocardiography 2009;26:489-494.

5. Abe K, Toba M, Alzoubi A, Ito M, Fagan KA, Cool CD, et al. Formation of plexiform lesions in experimental severe pulmonary arterial hypertension. Circulation 2010;121: 2747-2754.

6. Peter Dromparis, Roxane Paulin, Trevor H Stenson, Alois Haromy, Gopinath Sutendra and Evangelos D Michelakis. Attenuating endoplasmic reticulum stress as a novel therapeutic strategy in pulmonary hypertension. Circulation 2013:127: 115-125.

7. Wong CM, Bansal G, Pavlickova L, Marcocci L, Suzuki YJ. Reactive oxygen species and antioxidants in pulmonary hypertension. Antioxid Redox Signal 2013;18(14):1789-1796.

8. Aggarwal S, Gross CM, Sharma S, Fineman JR, Black SM. Reactive oxygen species in pulmonary vascular remodeling. Compr Physiol 2013;3(3):1011-1034.

9. Trachootham D, Alexandre J, Huang P. Targeting cancer cells by ROS-mediated mechanisms: a radical therapeutic approach? Nat Rev Drug Discov 2009; 8:579-591.

10. Hu HL, Zhang ZX, Chen CS, Cai C, Zhao JP, Wang X. Effects of mitochondrial potassium channel and membrane potential on hypoxic human pulmonary artery smooth muscle cells. Am J Respir Cell Mol Biol 2010;42: 661-666.

11. Wedgwood S, Dettman RW, Black SM. ET-1 stimulates pulmonary arterial smooth muscle cell proliferation via induction of reactive oxygen species. Am J Physiol 2001;281: L1058-L1067.

12. Nilsen-Hamilton $M$, Liu $Q$, Ryon J, Bendickson L, Lepont $P$, Chang $Q$. Tissue involution and the acute phase response. Ann NY Acad Sci 2003:995:94-108.

13. Wang Y, Lam KS, Kraegen EW, Sweeney G, Zhang J, Tso AW, et al. Lipocalin-2 is an inflammatory marker closely associated with obesity, insulin resistance, and hyperglycemia in humans. Clin Chem 2007;53:34-41.

14. DH Goetz, MA Holmes, N Borregaard, ME Bluhm, KN Raymond, RK Strong. The neutrophil lipocalin NGAL is a bacteriostatic agent that interferes with siderophore-mediated iron acquisition. Mol Cell 2002;10:1033-1043.

15. Roudkenar $\mathrm{MH}$, Halabian $\mathrm{R}$, Bahmani $\mathrm{P}$, Roushandeh $\mathrm{AM}$, Kuwahara $\mathrm{Y}$ Fukumoto M. Neutrophil gelatinase-associated lipocalin: a new antioxidant that exerts its cytoprotective effect independent on Heme Oxygenase-1. Free Radic Res 2011; 45(7):810-819.

16. Mehryar Habibi Roudkenar, Raheleh Halabian, Zahra Ghasemipour, Amaneh Mohammadi Roushandeh, Mahdi Rouhbakhsh, Mahin Nekogoftar, et al. Neutrophil gelatinase-associated lipocalin acts as a protective factor against $\mathrm{H}_{2} \mathrm{O}_{2}$ toxicity. Archives of medical research 2008:39:560-566.

17. Parisa Bahmani, Raheleh Halabian, Mehdi Rouhbakhsh, Amaneh Mohammadi Roushandeh, Nasser Masroori, Majid Ebrahimi, et al. Neutrophil gelatinase-associated lipocalin induces the expression of hemeoxygenase-1 and superoxide dismutase. Cell stress and chaperones 2010;15:395-403.
18. Lin $\mathrm{HH}$, Liao CJ, Lee YC, Hu KH, Meng HW, Chu ST. Lipocalin-2-induced cytokine production enhances endometrial carcinoma cell survival and migration. Int J Biol Sci. 2011;7(1):74-86.

19. Rodvold J, Mahadevan N, Zanetti M. Lipocalin 2 in cancer: when good immunity goes bad. Cancer Lett. 2012; 316(2):132-8.

20. Guoxiong $\mathrm{Xu}$, JinHee Ahn, SoYoung Chang, Megumi Eguchi, Arnaud Ogier, SungJun Han, et al. Lipocalin-2 induces cardiomyocyte apoptosis by increasing intracellular iron accumulation. J Biol Chem 2012;287:4808-4817.

21. I-Lun Hsin, Yueh-Chieh Hsiao, Ming-Fang Wu, Ming-Shiou Jang, Sheau-Chung Tang, Yu-Wen Lin, et al. Lipocalin 2, a new GADD153 target gene, as an apoptosis inducer of endoplasmic reticulum stress in lung cancer cells. Toxicology and applied pharmacology 2012;263:330-337.

22. Meng L, Liu X, Zheng Z, Li J, Meng J, Wei Y, Hu S. Original rat model of high kinetic unilateral pulmonary hypertension surgically induced by combined surgery. J Thorac Cardiovasc Surg 2013;146(5):1220-1226.

23. Liu X, Meng L, Shi Q, Liu S, Cui C, Hu S, Wei Y. Dermatopontin promotes adhesion, spreading and migration of cardiac fibroblasts in vitro. Matrix Biol 2013; 32(1):23-31.

24. Mari C Asensio-Lo' pez, Jesu' sSa' nchez-Ma' s, Domingo A Pascual-Figal, Sergio Abenza, et al. Involvement of ferritin heavy chain in the preventive effect of metformin against doxorubicin-induced cardiotoxicity. Free Radic Biol Med. 2013; 57(10):188-200.

25. Chen J, Baydoun AR, Xu R, Deng L, Liu X, Zhu W, et al. Lysophosphatidic acid protects mesenchymal stem cells against hypoxia and serum deprivation-induced apoptosis. Stem Cells 2008;26(1):135-45.

26. Shin EJ, Schram K, Zheng XL, and Sweeney G. Leptin attenuates hypoxia/reoxygenation-induced activation of the intrinsic pathway of apoptosis in rat $\mathrm{H} 9 \mathrm{c} 2$ cells. J Cell Physiol 2009;221:490-497.

27. Chakraborty S, Kaur S, Guha S, Batra SK. The multifaceted roles of neutrophil gelatinase associated lipocalin (NGAL) in inflammation and cancer. Biochim Biophys Acta 2012;1826(1):129-169.

28. Barrera C: Oxidative stress and lipid peroxidation products in cancer progression and therapy. ISRN Oncology 2012; : 137289.

29. Schmidt-Ott KM, Mori K, Kalandadze A, Li JY, Paragas N, Nicholas T, et al. Neutrophil gelatinase-associated lipocalin-mediated iron traffic in kidney epithelia. Curr Opin Nephrol Hypertens 2006;15:442-449.

30. Zap pitelli M, Washburn KK, Arikan AA, Loftis L, Ma Q, Devarajan P, et al. Urine neutrophil gelatinase-associated lipocalin is an early marker of acute kidney injury in critically ill children: a prospective cohort study. Crit Care 2007; 11:R84.

31. Fadel FI, Abdel Rahman AM, Mohamed MF, Habib SA, Ibrahim MH, Sleem $\mathrm{ZS}$, et al. Plasma neutrophil gelatinase-associated lipocalin as an early biomarker for prediction of acute kidney injury after cardio-pulmonary bypass in pediatric cardiac surgery. Arch Med Sci 2012; 8(2):250-255.

32. Jeffery TK, Morrell NW. Molecular and cellular basis of pulmonary vascular remodeling in pulmonary hypertension. Prog Cardiol Dis 2002;45:173-202.

33. Mandegar M, Fung YC, Huang W, Remillard CV, Rubin LJ, Yuan JX. Cellular and molecular mechanisms of pulmonary vascular remodeling: role in the development of pulmonary hypertension. Microvasc Res 2004;68:75-103.

34. Dorfmüller P, Perros F, Balabanian K, Humbert M. Inflammation in pulmonary arterial hypertension. Eur Respir J 2003:22:358-363. 